\title{
Papillary vs non-papillary access during percutaneous nephrolithotomy: Retrospective, match-paired case-control study
}

\author{
Ahmet Tahra ${ }^{1}$, Resul Sobay ${ }^{1}$, Ahmet Bindayi ${ }^{2}$, Ferhat Yakup Suceken ${ }^{1}$, Eyup Veli Kucuk ${ }^{1}$ \\ ${ }^{1}$ Department of Urology, Health Sciences University, Umraniye Teaching Hospital, Istanbul, Turkey; \\ 2 Department of Urology, Burhan Nalbantoglu Hospital, Cyprus.
}

\begin{abstract}
Summary Objective: The most crucial steps of percutaneous nephrolithotomy (PCNL) are the percutaneous access and dilation of the access route. Recent literature suggests that papillary access to renal calyx is the accepted method. Despite this rule, we do not always make papillary puncture and we puncture wherever we can to achieve stone-free status and reduce unnecessary access. In this study, we present our results with papillary vs non-papillary access in patients with a kidney stone.

Material and methods: Two hundred and seven patients with non-papillary access and 69 patients with papillary access who had similar demographics (age, body mass index (BMI), stone size) were selected with pair match analysis (3:1).

Preoperative and postoperative data were collected from the patient's chart. Operative time (from starting surgery to nephrostomy tube), drop-in hematocrit level, transfusion rate, duration of hospital stay, perioperative and postoperative complications (Clavien-Dindo Classification) and stone-free status (no or $<3 \mathrm{~mm}$ residual stone) were also evaluated in both groups.

Results: The mean operative time was similar in between two groups. The mean hematocrit decreases not differ between the two groups $(p=0.56)$. In papillary group, only 2 patients (3.2\%) required transfusion and only one patient (1.4\%) in the non-papillary group had a transfusion with no statistically significant difference $(p=0.43)$. The overall complication rates were $7.1 \%$ in the papillary group and $7.2 \%$ in the non-papillary group $(p=0.89)$. Postoperative mean creatinine level was similar between the two groups.

Conclusions: In this study, we found that non-papillary access is a feasible option for PCNL in the terms of stone-free status and complication rates.
\end{abstract}

KEY WORDS: Percutaneous nephrolithotomy; Access; Papillary; Non-papillary.

Submitted 14 September 2019; Accepted 13 November 2019

\section{INTRODUCTION}

Percutaneous nephrolithotomy (PCNL) is still the standard therapy for the larger calculi in the kidney (1). The most crucial steps of PCNL are the percutaneous access and dilation of the access route. As accepted by literature, punctures must be done through the papilla of the posterior renal calyx to avoid the major vascular structures of the kidney (2).

Despite this rule, access through the papilla is not always achievable. In our huge volume percutaneous surgery centre (over 400 cases per year) we do not always make papillary puncture and we puncture wherever we can to achieve stone-free status and reduce unnecessary access. Non-calyceal puncture method was recently published and the authors concluded that it is feasible and probably not as dangerous as it was stated (3). In this study, we present our results with papillary vs non-papillary access in patients with kidney stone.

\section{MATERIAL AND METHODS}

After an Ethics Committee approval was obtained, a total of 638 patients patient undergoing PCNL between January 2017 and June 2018 were analyzed. Two hundred and seven patients with non-papillary access and 69 patients with papillary access who had similar demographics (age, BMI, maximum diameter of the stone) were selected with pair match analysis (3:1). Preoperative and postoperative data were collected from the patient's chart. Operative time (from starting surgery to nephrostomy tube), drop-in hematocrit level, transfusion rate, duration of hospital stay, perioperative and postoperative complications (Clavien-Dindo Classification) and stone-free status (no or $<3 \mathrm{~mm}$ residual stone) were also evaluated in both groups. Preoperative and postoperative third-month creatinine level were also recorded and analyzed. Followup was made with low dose non-enhanced computerized tomography (NECT) three months after surgery.

Patients with a solitary kidney, history of previous surgery (open or endoscopic) or Extracorporeal Shock Wave Lithotripsy (ESWL) for the same kidney, congenital anatomical variants (horseshoe kidney, ectopic kidney) were excluded.

In surgical technique; after insertion of open-end $4 \mathrm{~F}$ ureteral catheter in lithotomy position, patients were set to the prone position. All patients were treated with combined fluoroscopic and ultrasound guided PCNL. In papillary access group, papilla of the calyx was punctured. In the other group, the puncture was made through infundibulum of the calyx. The puncture site was corrected under ultrasound control. After insertion of hydrophilic guidewire all cases were dilated up to 16 F with Amplatz dilators and then balloon dilatation was made up to $30 \mathrm{~F}$. Rigid nephroscope and pneumatic lithotripter were used to remove calculi. Fragments were 
removed using basket catheter and forceps. At the end of the procedure, $14 \mathrm{~F}$ nephrostomy catheter was inserted in all cases.

Statistical analysis was performed with the IBM SPSS version 20 (IBM Corp., Armonk, NY, USA). Fischer's exact test, Pair-Match analysis, Mann-Whitney U, t-test and were used for the analysis of the data and statistical significance was accepted as $\mathrm{p}$-value $<0.05$.

\section{Results}

Patient age, BMI and stone size were similar (nearly the same) in both groups according to case match analysis (Table 1). The mean operative time was also similar. The average of hematocrit decrease was $3.45 \pm 2.2$ in the papillary group and $3.89 \pm 3.3$ in the non-papillary group with no difference between the two groups $(\mathrm{p}=0.56)$.

In the papillary group, only 7 patients $(3.8 \%)$ required transfusion and only one patient (1.4\%) in the non-papillary group had a transfusion with no statistically significant difference $(\mathrm{p}=0.43)$.

The overall complication rates were similar being $7.1 \%$ in the papillary group and $7.2 \%$ in the non-papillary group $(\mathrm{p}=0.89)$. According to Clavien-Dindo Classification, only one patient in the non-papillary group and three patients in non-papillary group had Class III ${ }^{a}$ (required selective angioembolization because of uncontrolled bleeding) complication ( $\mathrm{p}=0.87$ ).

The mean duration of hospital stay was also similar. Postoperative mean creatinine level was similar between the two groups. All statistical analysis are showed in Table 2.

\section{Table 1.}

Patient characteristics.

\begin{tabular}{|lccc|}
\hline & $\begin{array}{c}\text { Papillary } \\
\text { group (n: 207) }\end{array}$ & $\begin{array}{c}\text { Non-papillary } \\
\text { group (n: 69) }\end{array}$ & p value \\
\hline Age & $51.6 \pm 13.54$ & $52.2 \pm 12.43$ & 0.94 \\
\hline BMl $\left(\mathrm{kg} / \mathrm{m}^{2}\right)$ & $28.3 \pm 5.2$ & $27.9 \pm 3.6$ & 0.93 \\
\hline Stone size $(\max$ diameter, $\mathrm{cm})$ & $2.46 \pm 4.6$ & $2.38 \pm 5.1$ & 0.85 \\
\hline Gender $\mathrm{F} / \mathrm{M}, \mathrm{n}$ & $66 / 141$ & $24 / 45$ & \\
\hline Location & & & \\
Upper & 23 & 9 & \\
Middle & 25 & 11 & \\
Pelvis & 82 & 26 & \\
Lower & 77 & 23 & \\
Values are presented as mean values \pm standard deviation. & & \\
BMl $=$ body mass index; $M=$ male; $F=$ female. & & \\
\hline
\end{tabular}

\section{Table 2.}

Perioperative and postoperative outcomes and complications.

\begin{tabular}{|lccc|}
\hline & $\begin{array}{c}\text { Papillary } \\
\text { group (n: 207) }\end{array}$ & $\begin{array}{c}\text { Non-papillary } \\
\text { group (n: 69) }\end{array}$ & p value \\
\hline Operation time, min & $58.3 \pm 14.3$ & $56.8 \pm 15.3$ & 0.56 \\
\hline Drop in hematocrit level & $3.45 \pm 2.2$ & $3.43 \pm 2.7$ & 0.93 \\
\hline Length of hospital stay, days & $4.45 \pm 1.9$ & $4.51 \pm 1.8$ & 0.42 \\
\hline Stone Free Status (\%) & $86.4 \%$ & $85.5 \%$ & 0.66 \\
\hline Mean change in Creatinine, $\mathrm{mg} / \mathrm{dL}$ & $0.06 \pm 0.29$ & $0.05 \pm 0.41$ & 0.68 \\
\hline Overall complications, (\%) & 7.1 & 7.2 & 0.89 \\
$\geq$ Clavien-Dindo Class III & 1.4 & 1.4 & 0.87 \\
\hline Transfusion rate (\%) & 3.8 & 1.4 & 0.16 \\
\hline Values are presented as mean values \pm standard deviation. & & \\
\hline
\end{tabular}

\section{Discussion}

After Fernstrom and Johansson reported the first percutaneous nephrolithotomy in 1976 and Alken et al. published the first series of percutaneous interventions for removing renal stones, PCNL started to become widely used for renal stones $(4,5)$. Operative technique and endoscopic equipment are still evolving to increase success and decrease morbidity.

Recent literature suggests that preferred puncture site is papillary access on the avascular line to avoid the risk of bleeding. Sampaio et al. were studied to determine the best route to puncture in 1992 and they found that; in infundibular access, upper site was injured in $67.6 \%$ (41.1\% venous and $26.5 \%$ arterial), mid site of kidney in $61.5 \%$ (38.4\% venous and $23.1 \%$ arterial) and lower site in $68.2 \%$ (54.6\% venous and $13.6 \%$ arterial).

In the direct puncture of pelvis $33.2 \%$ injuries were recorded but in calyceal fornix overall injuries were recorded only $7.7 \%$ and they were all venous injuries (6). This study had three major concerns; firstly, it was cadaveric study and renal-related anatomical tissue was not evaluated, secondly renal and cortical system was naive and anatomical changes due to stone was not considered, thirdly no renal functional evaluation was made, and recovery was not assessed.

Kallidonis et al. published a prospective randomized trial that compared papillary vs non-papillary access in PCNL, and they found that access to infundibulum is the feasible and safe procedure and it is not associated with higher blood loss and transfusion rate (7).

Kallidonis et al. evaluated infundibulum of the middle calyx approach technique by $99 \mathrm{mTc}$-dimercaptosuccinic acid SPECT/CT renal scintigraphies and/or computerized tomographies perfusion (CTP); they found that the punctures to the mid calyceal papilla fornix and infundibulum as well as pelvis have similar angles of approach and that effects on parenchyma involved in the tract dilation are similar (8).

They concluded that infundibular puncture can be an option to puncture in the performance of PCNL.

In another study Kyriazis et al. investigated the feasibility and safety of PCNL with non-calyceal access track; they operated 137 patients consecutively, including 10 cases with anatomical variations, under fluoroscopic guidance (3). Stone free status was $89.2 \%$ for a single stone, $80.4 \%$ with multiple and $66.7 \%$ for staghorn stones. The overall complication rate was $10.2 \%$ and the major complication rate was $3.6 \%$. The authors concluded that calyceal access is possible and safe with stone-free status and low complications.

The length of hospitalization is a new investigation era for PCNL. The technology continues to improve, and postoperative complications are decreasing, "outpatient" procedure is an option now for highly selected patients (9). However, we still routinely admitted patients to follow-up. In terms of hospital stay our results are comparable with a recent literature (10).

In this study, the overall complication rates were $7.1 \%$ for the papillary group and $7.2 \%$ for the non-papillary group. Transfusion rates were $3.8 \%$ and $1.4 \%$.

In a study by Wiesenthal et al. which compared shockwave lithotripsy, ureteroscopy, and PCNL for renal cal- 
culi between 100-300 $\mathrm{mm}^{2}$, they found that overall complication in PCNL was 14\% (11). In a recent study comparing retrograde intrarenal surgery versus PCNL, in PCNL group bleeding rate was $6.87 \%$ and it was higher than our results (10).

This study has several limitations; firstly, the study has a retrospective nature. Secondly, control patients were not randomized and selected for analysis. The surgeons (three surgeons) are very experienced - over 300 cases and that may cause low complication rates. Patients are not consecutively enrolled in this study and anatomical variations are not evaluated. Multiple access and longterm complications are not also evaluated. Low number of patients because of the wide exclusion criteria is another limitation of this study.

\section{Conclusions}

In this study, we found that non-papillary access is a feasible option for PCNL in terms of stone-free status and complication rates. More anatomical and radiological, prospective randomized studies involving a great number of patients may be needed to determine the safety and efficacy of non-papillary access.

\section{REFERENCES}

1. Turk C, Petrik A, Sarica K, et al. EAU Guidelines on Interventional Treatment for Urolithiasis. Eur Urol. 2016; 69:475-482.

2. Gupta M. OM, Shah JB, McDougall EM, Smith, A. Percutaneous management of the upper urinary tract. Campbell-Walsh Urology. ninth ed. Saunders Elsevier, Philadelphia, PA. 2007.

3. Kyriazis I, Kallidonis P, Vasilas M, et al. Challenging the wisdom of puncture at the calyceal fornix in percutaneous nephrolithotripsy: feasibility and safety study with 137 patients operated via a noncalyceal percutaneous track. World J Urol. 2017; 35:795-801.

4. Fernstrom I, Johansson B. Percutaneous pyelolithotomy. A new extraction technique. Scand J Urol Nephrol. 1976; 10:257-259.

5. Alken P, Hutschenreiter G, Gunther R, Marberger M. Percutaneous stone manipulation. J Urol. 1981; 125:463-466.

6. Sampaio FJ, Zanier JF, Aragao AH, Favorito LA. Intrarenal access: 3-dimensional anatomical study. J Urol. 1992; 148:1769-1773.

7. Kallidonis P, Kyriazis I, Kotsiris D, et al. Papillary vs nonpapillary puncture in percutaneous nephrolithotomy: a prospective randomized trial. J Endourol. 2017; 31:S4- S9.

8. Kallidonis P, Kalogeropoulou C, Kyriazis I, et al. Percutaneous nephrolithotomy puncture and tract dilation: evidence on the safety of approaches to the infundibulum of the middle renal calyx. Urology. 2007; 107:43-48.

9. Wei C, Zhang Y, Pokhrel Gm et al. Research progress of percutaneous nephrolithotomy. Int Urol Nephrol. 2018; 50:807-817.

10. Zheng C, Xiong B, Wang Hm et al. Retrograde intrarenal surgery versus percutaneous nephrolithotomy for treatment of renal stones > 2 cm: a meta-analysis. Urol Int. 2014; 93:417-424.

11. Wiesenthal JD, Ghiculete D, D'A Honey RJ, Pace KT. A comparison of treatment modalities for renal calculi between 100 and $300 \mathrm{~mm}$ 2: are shockwave lithotripsy, ureteroscopy, and percutaneous nephrolithotomy equivalent? J Endourol. 2011; 25:481-485.

\section{Correspondence}

Ahmet Tahra, MD (Corresponding Author)

ahmettahra@gmail.com

Resul Sobay, MD

drresulsobay@gmail.com

Ferhat Yakup Suceken, MD

ykpsckn@gmail.com

Eyup Veli Kucuk, MD

eyupveli@gmail.com

Department of Urology, Health Sciences University

Umraniye Teaching Hospital, Istanbul, Turkey

Adem Yavuz Cad. No: 134766 Istanbul (Turkey)

ORCID ID: 0000-0002-5158-5630

Ahmet Bindayi, $M D$

ahmetbindayi@gmail.com

Department of Urology, Burhan Nalbantoglu Hospital, Cyprus 